\title{
Mpemba Effect Demystified
}

Ren Tier

\begin{abstract}
The Mpemba Effect (ME) is known ${ }^{1}$ as the counter intuitive effect of faster freezing of a beaker of warm water than the same but colder sample, under apparently same conditions (e.g. in the freezer, Aristotle will have done it outdoor).

The astonishment about this misled all the investigations and explanations intuitively into looking for water/ice/solidification properties, and the key, the heat transfer, was not adequately taken into account.

If we trust classical physics and energy conservation, faster freezing of water must be primarily associated with a greater heat flow, not with mystical water properties or behavior.
\end{abstract}

It is shown that the trivial conductivity through the bottom of the beaker adequately explains the ME.

(ME reloaded: why can colder water sample freeze slower than warmer one? :-)

\section{Previous investigations}

Numerous publications on ME describe and discuss: super-cooling, evaporation, dissolved salts and gases, molecular or quantum mechanical "memory" effects, etc. Only a few rough details are given on the size and shape of the refrigerator, cups, cooling power, cooling surfaces, placement in the freezer, materials, etc.

It looks like mind has been distracted by sham of ME.

1 a) not meant is the effective pouring of boiling water into frosty air

b) Possibly the term ME is used in physics as a "terminus technicus" for similar effects. The author lacks in-depth knowledge of this.

(scary sounding reports like "Exponentially Accelerated Approach to Stationarity in Markovian Open Quantum Systems through the Mpemba Effect") 


\section{Indication of heat exchange}

Remarkable are the typical plots of the cooling with ME emerging. The blue curve of the cold sample was moved next to the red curve (warm sample) with the purpose to point out the different curves in the same temperature range traversed by both, $\sim 18 \rightarrow 0^{\circ} \mathrm{C}$.

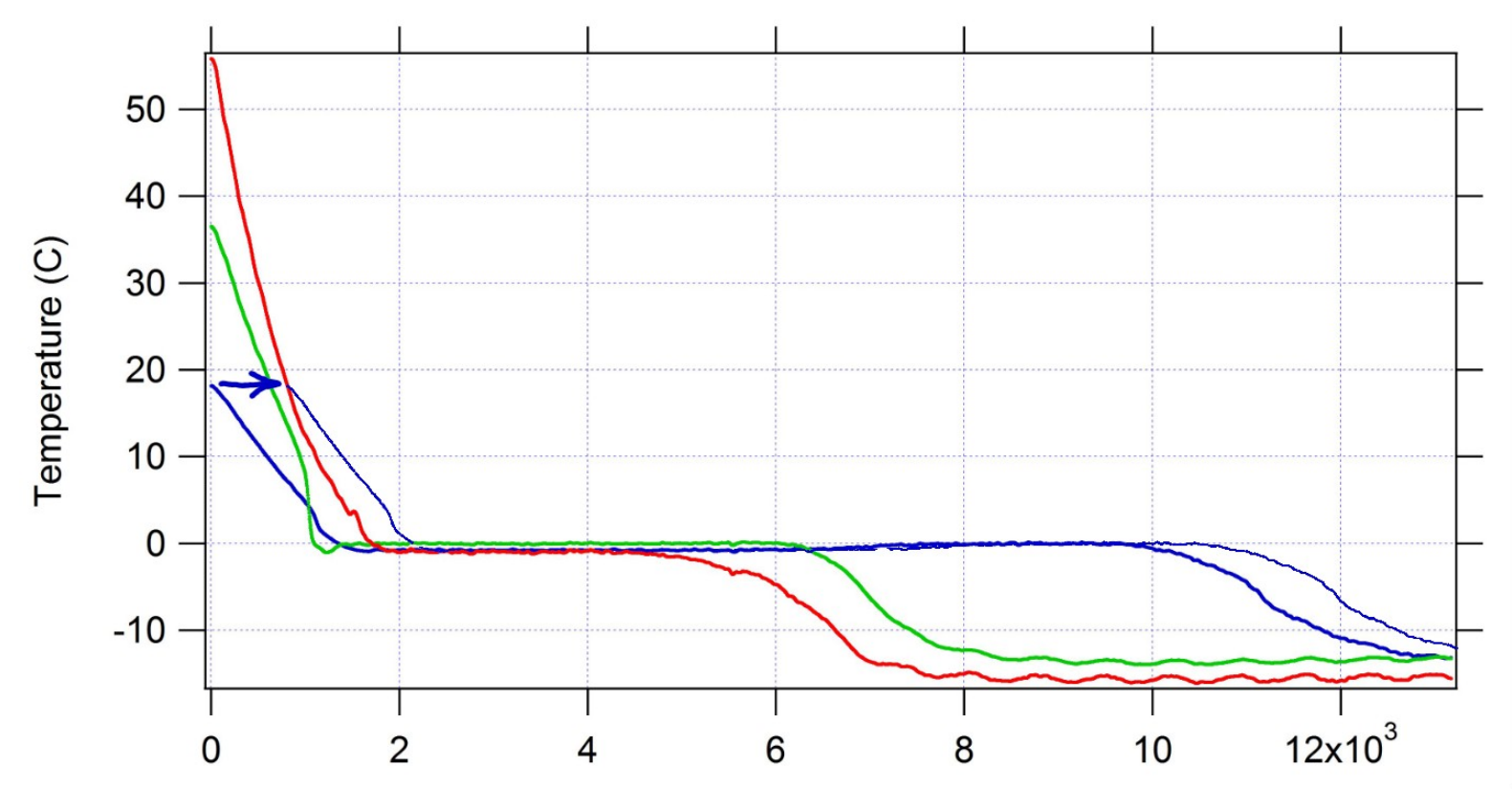

Fig. 1. from [1]

The intensive cooling of the warm sample (red) takes place already in the positive temperature range, even at temperatures that the cold sample passes through (notice temperature gradient), and it looks like it continues at $0^{\circ} \mathrm{C}$ and below The faster freezing is the result of stronger heat dissipation.

Nothing mystic about it.

The faster freezing and the intuitive but false feeling that the bottom of the glass beaker and the layer of ice underneath would be negligible and have rather some insulating properties, obfuscate the root cause. 


\section{Thesis:}

\section{ME is caused by conduction through the bottom of the beaker}

The search for an explanation of the ME through natural convective flow was not successful (Appendix A2). The conduction through the bottom of the beaker, on the other hand, is very promising:

- With the first intuitive approach, glass and ice are often perceived as having poor thermal conductivity and are therefore in ME context negligible.

Compared to the free air cooling, this is a mistake. A rough estimation is calculated in Appendix A1, just to check the plausibility of the thesis.

- It is obvious that a warm sample melts the surface of the bottom ice in the freezer and thus creates a good heat transfer, whereas a cold sample possibly does not or not so good.

- Let us now turn attention to the boundary layer between glass and ice.

In a simulation (some details in Appendix A3) is shown the temperature curve over time near the boundary layer.

These simulations do not claim to depict reality. They serve to rough illustrate the thesis.

In Fig. 2, the lower curve, we can see that in the colder sample $\left(\sim 20^{\circ} \mathrm{C}\right)$ the temperatures remain below $0^{\circ} \mathrm{C}$ just below the surface, i.e. the upper layer of ice can hardly thaw. The bottom contact remains limited to few support points, i.e. poor heat transfer.

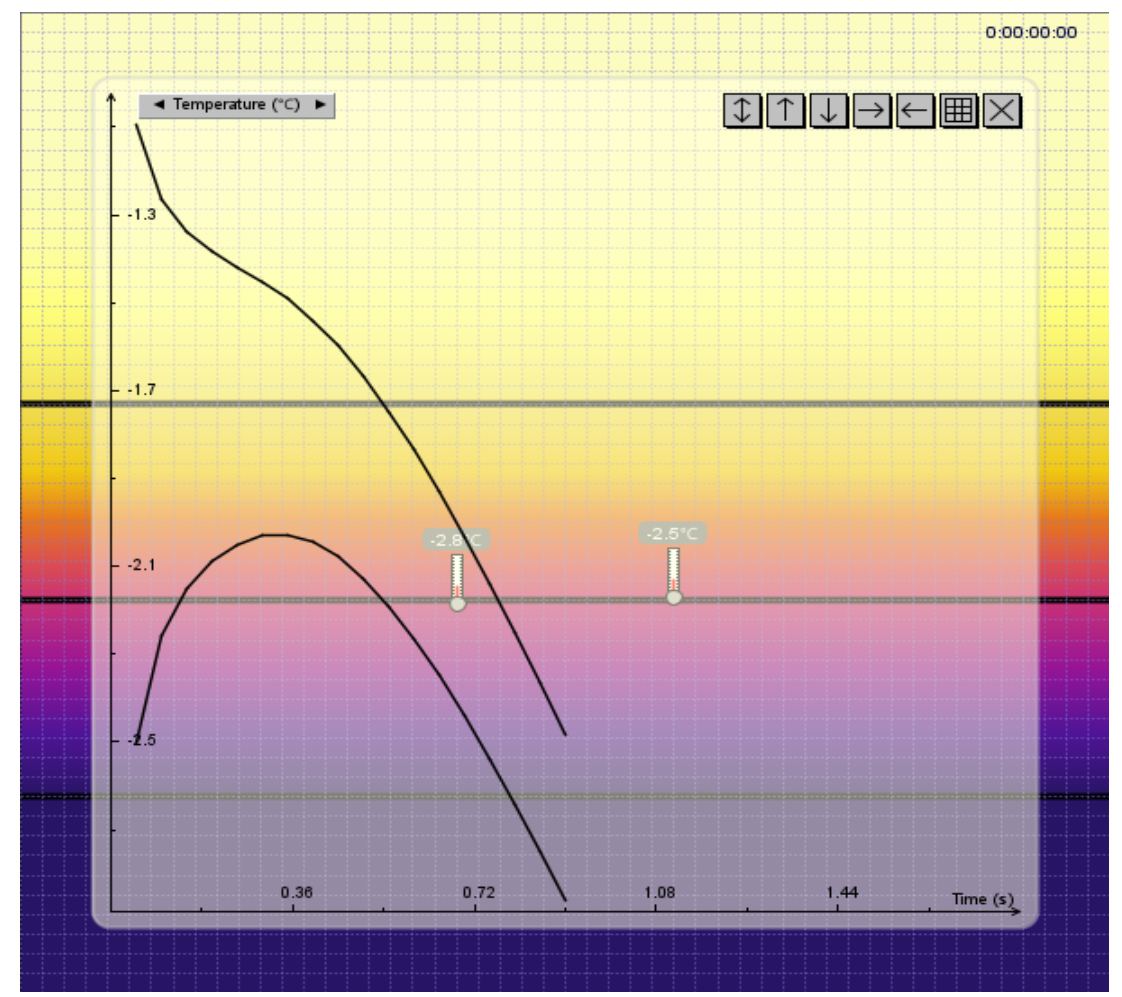

Fig. 2. Simulation: Temperatures near the boundary layer between ice and glass, $20^{\circ}$ 
The trivial case, in which a warm sample at the beginning melts the ice, is presented here in order to estimate the dynamics.

The melting process is not simulated. Positive temperatures in the ice layer show that the ice would melt. After freezing again, the energy balance and the temperature should be plausible again.

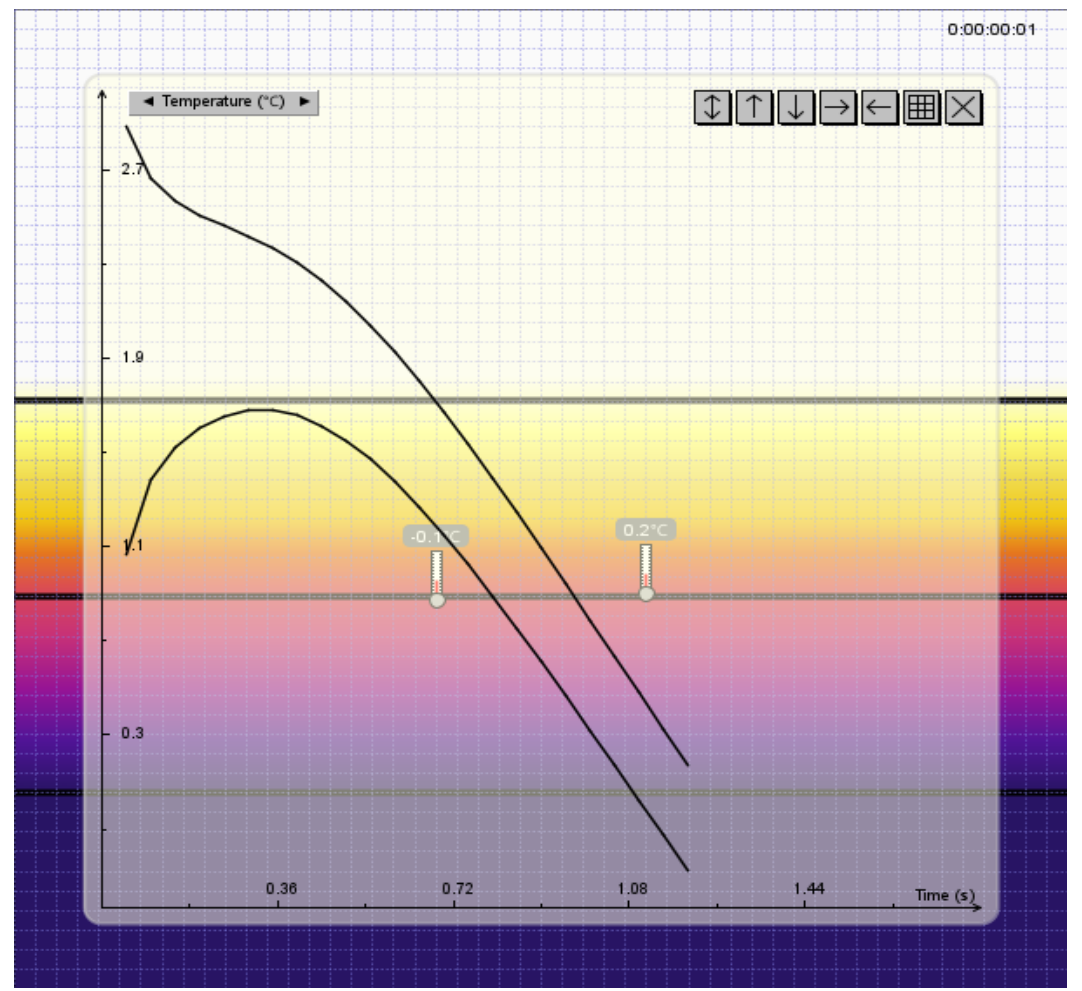

Fig. 3. Simulation: Temperatures near the boundary layer between ice and glass, $30^{\circ}$ C

- Dependency ME on the sample temperature and cup shape:

Laboratory beakers have a somewhat uneven bottom and only partial contact with the hoar frost or ice surface. It is therefore also plausible that a warmer glass bottom melts the ice or hoar frost in the cooling compartment more and more deeply, forming a larger film of water, which leads to a sustained better heat / cold exchange even after freezing.

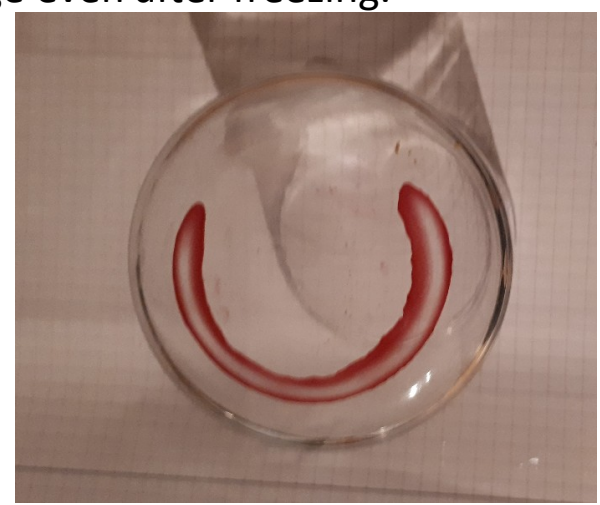

Fig. 4. Partial wetting of a beaker bottom on a glass pane. (just to illustrate the thesis) 
With a flat glass bottom and flat cooling surface, even the smallest amounts of water can wet out well and significantly facilitate heat transfer. On other hand, with clean cooling surface and uneven beaker no good thermal contact results.

- The known poor repeatability of the ME can be explained by the fact that after the warm beaker was put down it was moved (after a few seconds when the beaker already sticks in fridge) and the bottom glass-ice connection was unconsciously and unrecognized destroyed.

- Possibly (was not further investigated) the container material affects ME. A laboratory beaker made of borosilicate glass has low thermal expansion, which means that the connection between ice and glass does not break off under thermal stresses.

- It is not sufficient to consider only the cooling times until freezing begins. During freezing and faster cooling, the formerly warm sample gains more time, the longer the process lasts. This gain in time must "sponsor" the first phase of cooling down from the warmer state. This can also be clearly seen in Fig. 1 and Fig. 5. For quantitative results can/should be taken a negative temperature limit from the middle of the sample.

Furthermore we can early see forthcoming ME on the temperature gradient.

- We expect that a higher water level in the sample, i.e. a larger sample, increases the total time of the freezing process in favor of the warmer sample (see above). (side and top insulation should have similar effect)

- To get good evidence it is important for cold sample that the fridge/ice has sufficient low temperature to overwhelm glass temperature to prevent melting and sticking afterwards. The appropriate balance of temperatures with given thermal diffusivity of ice vs. glass are essential.

- A good indication for emerging of ME (warm sample) should be distinct sticking of the beaker to the fridge bottom (and easy sliding of cold sample).

- Freezing a sample not on the cooling surface or mass (e.g. in a drawer) makes emerging ME more difficult.

- Decreasing ice thickness under the warm sample (distance to the coolant) is likely to have a somewhat helping effect on ME. On the other hand, ME can arise on an ice block (not further investigated). 


\section{Verification / plausibility check in experiment}

Although the thesis itself is convincing, finally some experiments.

Measurements were taken as they came, without selection or optimization.

They are largely plausible.

(3sig or 6sig evidence was not aimed for ... ;-).

Used was a cheap BMP280 with ESP8266 controller. The values were slightly offset afterwards $\left(0.7 \rightarrow 0^{\circ} \mathrm{C}\right.$ freezing)

Beaker $\mathrm{D}=60 \mathrm{~mm}, \mathrm{H}=85, \mathrm{~m}=75 \mathrm{~g}, 150 \mathrm{ml}$ Water ( $3 / 4$ full)

The samples were put in into fridge always at about lowest temperature, just after the compressor pauses (monitored simply with NTC thermistor; my fridge has long cycle and big temperature hysteresis $-20-10^{\circ} \mathrm{C}$ )

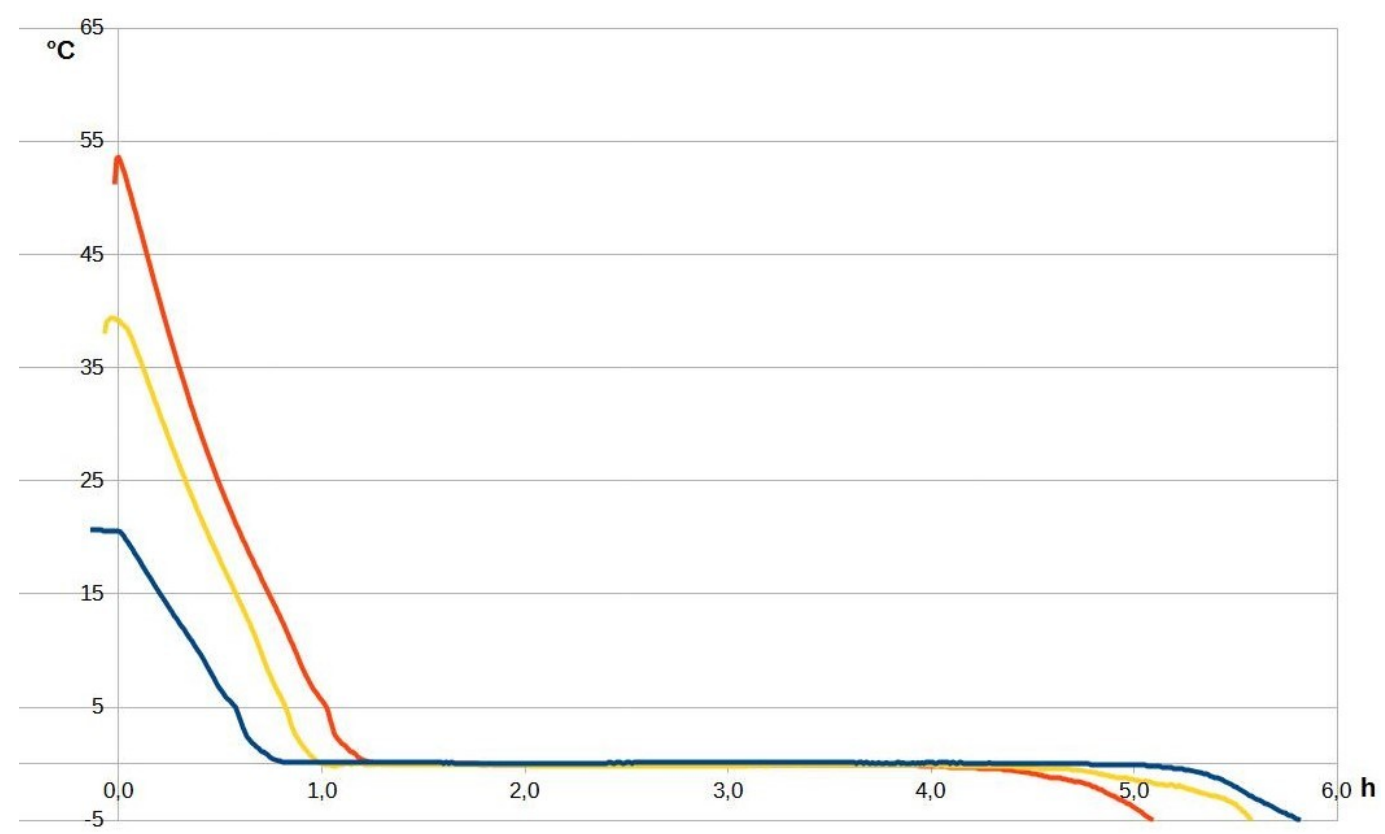

Fig. 5. Measurements

few, but IMO there is no need to exercise it further 


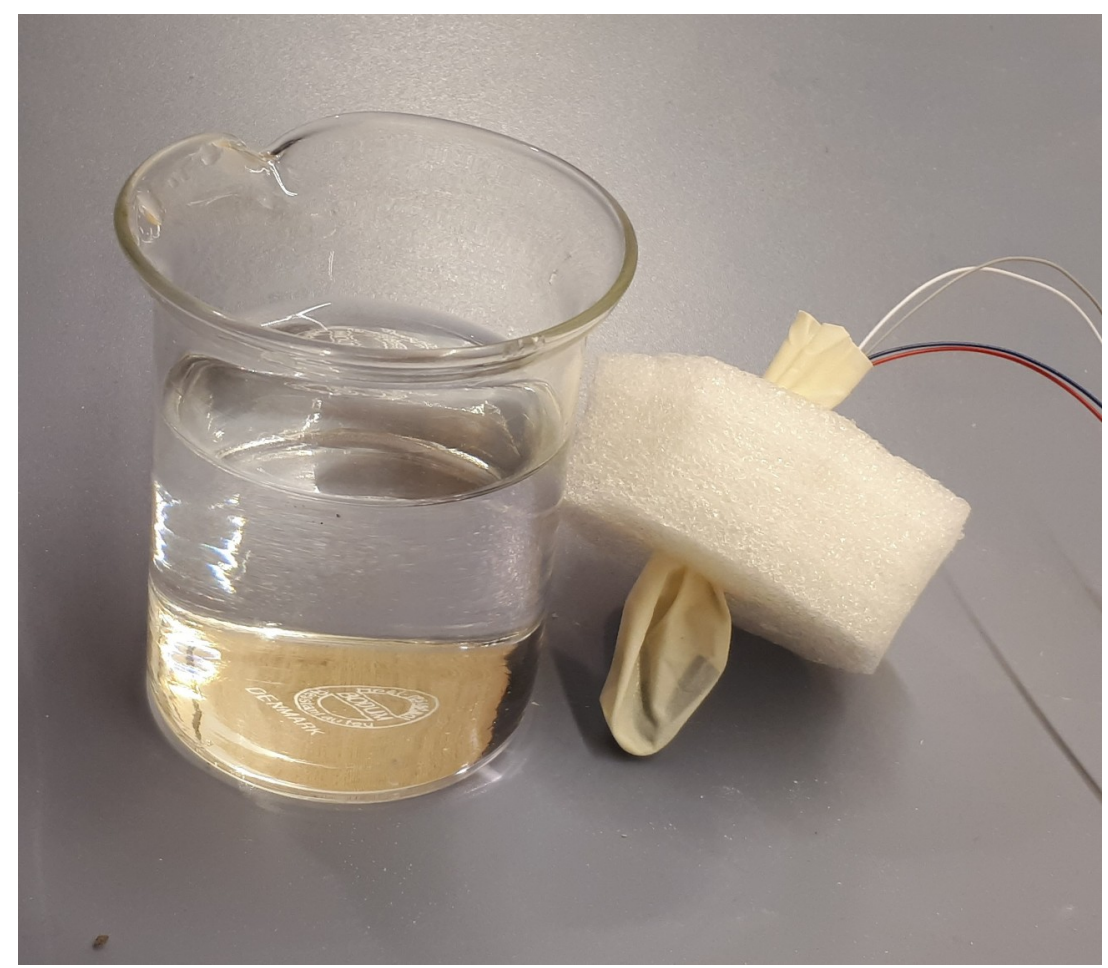

Fig. 6. Equipment

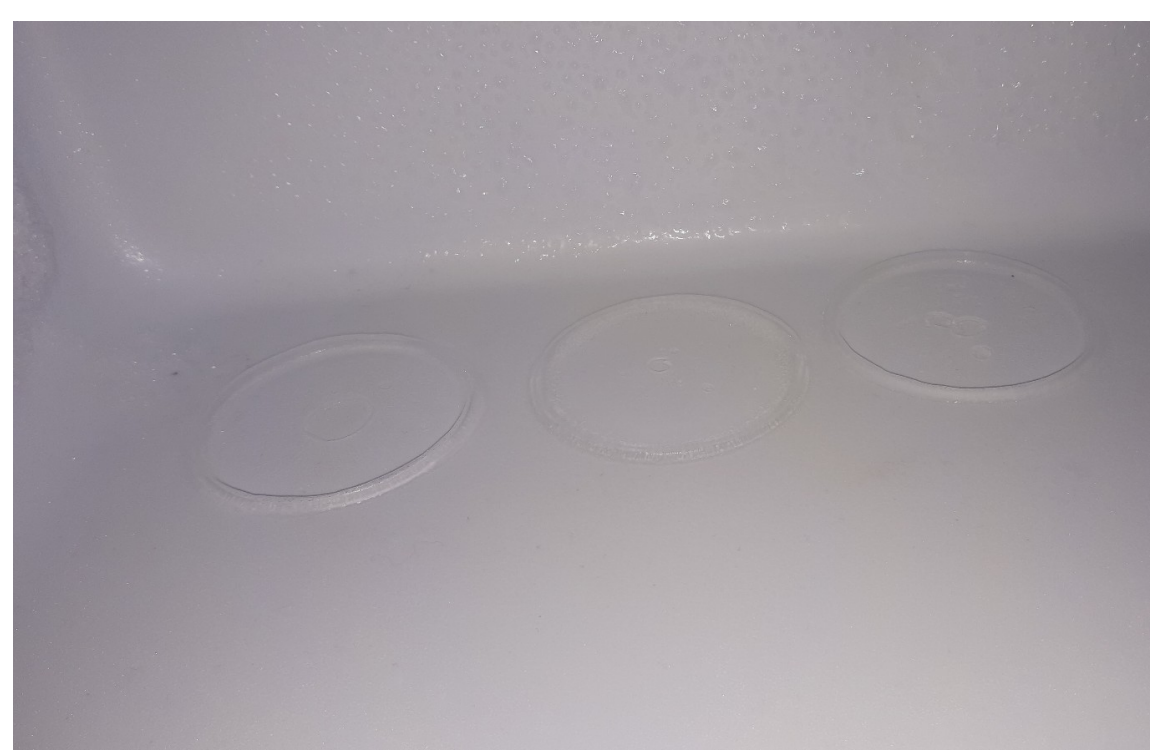

Fig. 7. Marks from warm samples in fridge 


\section{Counter example (ME avoided)}

For verification purposes should be possible to induce and control quickly freezing a cold samples, and warm samples slowly.

- The cold sample was put on wet piece of paper towel, to ensure good bottom contact.

- The warm sample was displaced after cooling to just above $0^{\circ} \mathrm{C}$.

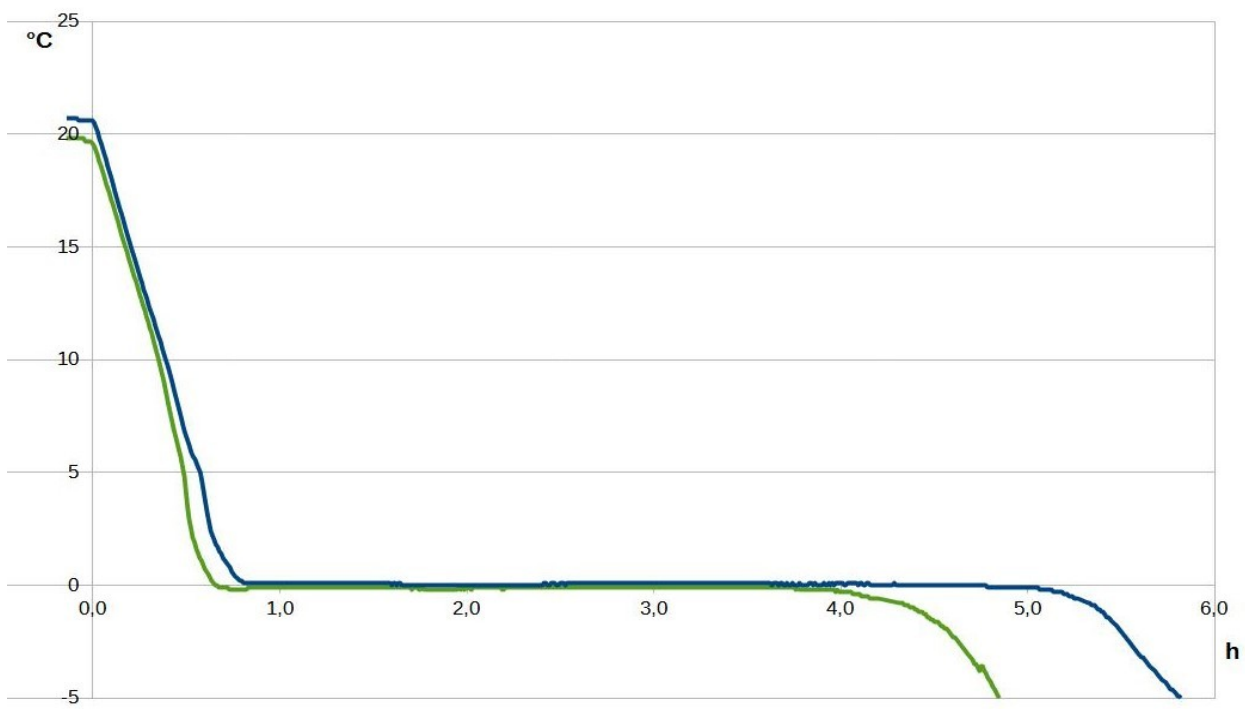

Fig. 8. Forced cooling i.e. wet placement of a cold sample (green).

The influence of the ice thickness must be considered, nevertheless there is strong cooling despite the increase in thickness (wet paper towel)

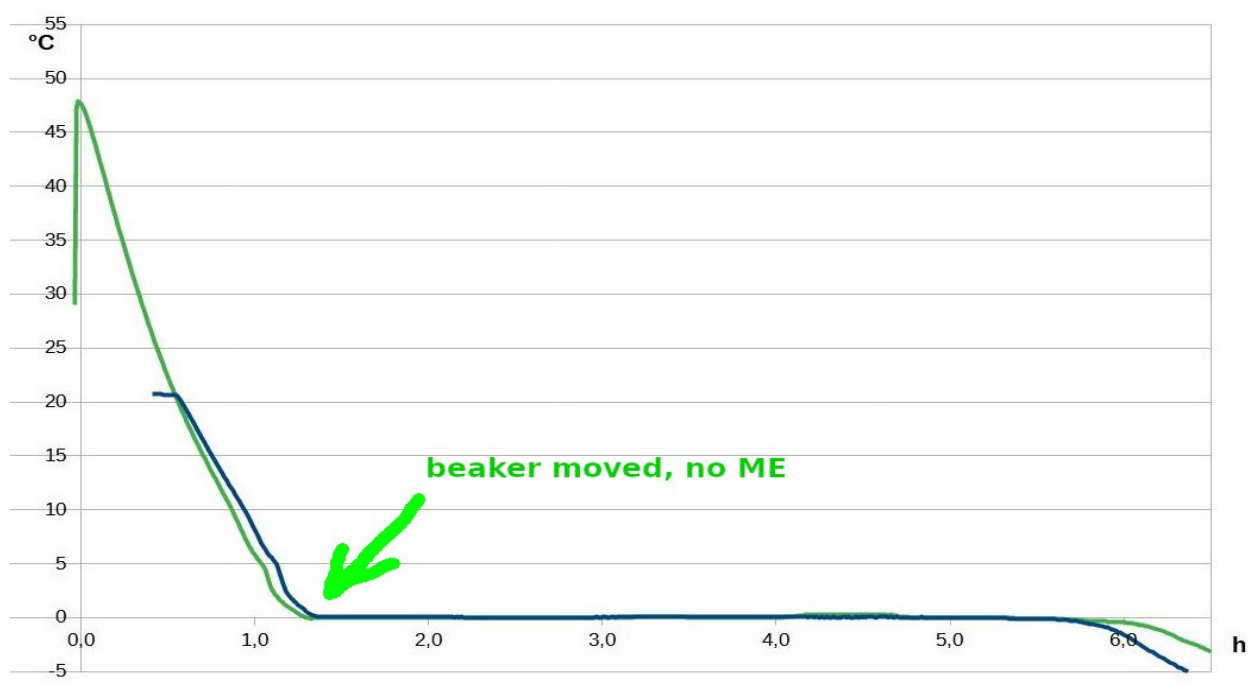

Fig. 9. Warm sample with ME broken at $0^{\circ} \mathrm{C}$ (green)

Supposed the sample was on the way to $\mathrm{ME}$, it was interrupted at $0^{\circ} \mathrm{C}$ moving the beaker. As expected freezing takes longer (however showing that warmer sample takes longer to cool is not an issue). 


\section{Conclusion:}

The thesis could easily be confirmed in experiment.

All that is simple, if you already understood.

It is much more difficult to break away from a preconceived or something that has been learned and found to be correct, than to grasp/learn anew. One sticks to one's old opinion and knowledge, and is reluctant to revise it.

Don't be a refusenik, think things to the end.

\section{REFERENCES}

[1] https://physics-archive.wooster.edu/JrlS/Files/Thomas Web Article.pdf 


\section{Appendix:}

\section{A1) Conduction through bottom of the beaker}

A rough estimate shows that a $1 \mathrm{~mm}$ layer of ice and a $50 \mathrm{~mm}$ diameter $1.5 \mathrm{~mm}$ thick boron-silicate glass pane (approx. Bottom $100 \mathrm{ml}$ laboratory beaker), $10^{\circ} \mathrm{C}$ temperature difference and ideal contact could transfer approx. $12 \mathrm{~W}(!)$ heat.

$$
\begin{aligned}
& \text { R_th }=\text { R_th_glas }+ \text { R_th_ice } \begin{array}{l}
\text { therm. resistance } \\
\text { heat flow (therm. power) }
\end{array} \\
& \begin{array}{l}
\mathrm{dQ} / \mathrm{dt}=\mathrm{dT} / R_{-} \text {th } \\
\mathrm{dQ} / \mathrm{dt}=10^{\circ} \mathrm{C} /\left(\begin{array}{l}
0.0015 \mathrm{~m} /(1.20 \mathrm{~W} / \mathrm{m}) / \mathrm{pi} / 0.025 \mathrm{~m}^{2}+ \\
\left.+0.001 \mathrm{~m} /(2.25 \mathrm{~W} / \mathrm{m}) / \mathrm{pi} / 0.025 \mathrm{~m}^{2}\right)=11.6 \mathrm{~W}
\end{array}\right.
\end{array}
\end{aligned}
$$

\section{A2) Notes on convective flow}

Convection was an approach to ME, that could not be confirmed.

Convection flows are subject to feedback effects (positive feedback, such as chimney effect; more flow, more heat exchange, even stronger flow) which make bi-stable states of heat transfer imaginable. According to this idea, it should be possible (water is not given a "memory" properties) to initiate the cooling of colder water in the jointly passed temperature range by initiating the faster "turbo cooling". Such effects were not observed. The author was unable to produce such effects, not even in flow simulation.

\section{Convection flows in the water:}

At water anomaly (approx. $4^{\circ} \mathrm{C}$ ) coming from both, warm and cold samples, the flow behavior is equalized. The switch of flow direction would break a possibly sustaining increased convection.

\section{Air convection}

The air in free convection is not efficient as a coolant. The air is known to be more an insulator.

The author has not succeeded in bringing up about dual flow states (with / without intensifying chimney-like flows or turbulence) nor simulating sufficiently strong persistent convection in a freezer-like system. 


\section{A3) Simulation}

The simulation was set up only as an estimate in order to support the thesis and does not claim to be exact.

The simulation was carried out with the Energy2D program (only 1D of interest). A simulation of the heat transfer from water, through a layer of glass, a layer of ice, to the cooling medium was carried out. Of primary interest is the time course of the temperature in the ice near the boundary layer to the beaker.

The $500 \times 500$ version of the program was used, within $5 \times 5 \mathrm{~mm}$ boundaries, i.e. resolution of $0.01 \mathrm{~mm}$

The following 4 layers were parameterized:

from top

- $2 \mathrm{~mm}$ water, with different temp, e.g. $+20^{\circ} \mathrm{C}$

- $1 \mathrm{~mm}$ borosilicate glass $1 \mathrm{~mm}$ with temp like water

- $1 \mathrm{~mm}$ ice at temp- $15^{\circ} \mathrm{C}$

- $1 \mathrm{~mm}$ fixed temperature $-15^{\circ} \mathrm{C}$ (cooler)

- The temperature was taken in the top layer of ice and the bottom layer of glass $(0.01 \mathrm{~mm})$

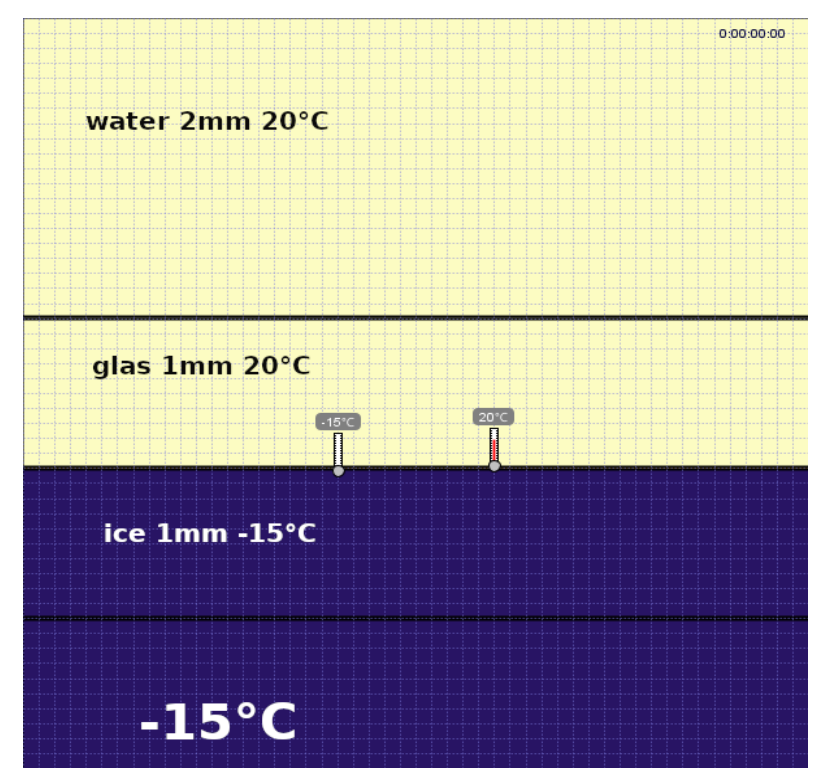

Figure 5. Simulation setup, initial state 